\title{
Unsupervised Domain Adaptation for Static Malware Detection based on Gradient Boosting Trees
}

\author{
Panpan Qi \\ School of Computing \\ National University of Singapore \\ qipanpan@u.nus.edu \\ Lei Zhu \\ NUS Graduate School \\ Integrative Sciences and Engineering Programme \\ e0203764@u.nus.edu
}

\author{
Wei Wang \\ School of Computing \\ National University of Singapore \\ wangwei.cs@gmail.com \\ See Kiong Ng \\ Institute of Data Science \\ National University of Singapore \\ seekiong@nus.edu.sg
}

\begin{abstract}
Static malware detection is important for protection against malware by allowing for malicious files to be detected prior to execution. It is also especially suitable for machine learning-based approaches. Recently, gradient boosting decision trees (GBDT) models, e.g., LightGBM (a popular implementation of GBDT), have shown outstanding performance for malware detection. However, as malware programs are known to evolve rapidly, malware classification models trained on the (source) training data often fail to generalize to the target domain, i.e., the deployed environment. To handle the underlying data distribution drifts, unsupervised domain adaptation techniques have been proposed for machine learning models including deep learning models. However, unsupervised domain adaptation for GBDT has remained challenging. In this paper, we adapt the adversarial learning framework for unsupervised domain adaptation to enable GBDT learn domain-invariant features and alleviate performance degradation in the target domain. In addition, to fully exploit the unlabelled target data, we merge them into the training dataset after pseudo-labelling. We propose a new weighting scheme integrated into GBDT for sampling instances in each boosting round to reduce the negative impact of wrongly labelled target instances. Experiments on two large malware datasets demonstrate the superiority of our proposed method.
\end{abstract}

\section{CCS CONCEPTS}

- Computing methodologies $\rightarrow$ Transfer learning; • Security and privacy $\rightarrow$ Malware and its mitigation.

\section{KEYWORDS}

unsupervised domain adaptation, malware detection, gradient boosting decision tree

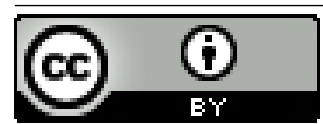

This work is licensed under a Creative Commons Attribution International 4.0 License.

CIKM '21, November 1-5, 2021, Virtual Event, QLD, Australia

(C) 2021 Copyright held by the owner/author(s).

ACM ISBN 978-1-4503-8446-9/21/11.

https://doi.org/10.1145/3459637.3482400

\section{ACM Reference Format:}

Panpan Qi, Wei Wang, Lei Zhu, and See Kiong Ng. 2021. Unsupervised Domain Adaptation for Static Malware Detection based on Gradient Boosting Trees. In Proceedings of the 30th ACM International Conference on Information and Knowledge Management (CIKM '21), November 1-5, 2021, Virtual Event, QLD, Australia. ACM, New York, NY, USA, 10 pages. https: //doi.org/10.1145/3459637.3482400

\section{INTRODUCTION}

Malware is one of the big threats to cyber security. Recently, machine learning models have been applied for malware detection [1, 34]. Specifically, gradient boosting decision trees (GBDT) [13] have achieved the state-of-the-art performance for detecting and classifying malware in static analysis [1]. Static analysis is important for protection against malware as it allows for the malicious files to be detected prior to execution. Unlike dynamic analysis which requires running the malware (in controlled environment) to collect behavioral information for classification, static malware detection directly analyses the binary of an input software program to determine if it is malicious or not, using hand-crafted features or more practically, machine-learned classifiers. However, GBDT and other machine learning models have a common assumption that the test data are drawn from the same distribution as the training data [2]. This assumption fails in the malware detection task because malware evolves rapidly over time [3]. As a result, malware detection models trained on labelled data, called source domain, often face performance degradation issues on test data, called target domain, due to the underlying distribution drifts. Therefore, it is important to adapt the model from the source domain to the target domain.

Unsupervised domain adaptation (UDA) techniques have been proposed to deal with the distribution drift issue between the labelled source data and unlabelled target data. Current approaches of UDA can be divided into two main categories. The first category consists of importance weighting approaches that reweight samples from the source domain to match the distribution of target domain $[16,18]$, while the approaches in the second category aim to find a common feature space in which the features are invariant across the domains [24, 27]. Most of the recent research works on UDA belong to the second category and are conducted on deep learning methods because deep neural networks (DNNs) can learn transferable features [30] via the layer abstraction. For example, $[14,26]$ leverage adversarial learning to train a domain 
discriminator and adapt features extracted by DNNs to confuse the discriminator alternatively so that the resulting learned features are domain-invariant. However, while end-to-end DNNs had shown great successes dealing with homogeneous data such as pixels in images and words in text, they do not do well for malware detection in which the data consist of complex and heterogeneous raw features. For example, EMBER [1] involves the extraction of a 2351dimension vector consisting of eight heterogeneous groups of raw features for a Windows portable executable (PE) file. We think this is one major reason that state-of-the-art end-to-end DNNs were easily outperformed by LightGBM [17], an out-of-the-box GBDT implementation, on the malware dataset and others $[4,20]$.

It is non-trivial to apply existing UDA techniques into GBDT for malware detection. Directly applying the importance weighting approaches $[16,18]$ does not leverage the properties of GBDT, given that GBDT internally generates the weights for instances as well, which may conflict with the importance weight generated by the UDA methods. The more recent UDA methods, designed for representation learning methods such as DNNs based on learning domain-invariant features $[14,26]$, are also not directly applicable as GBDT models do not learn features explicitly.

In this paper, we design features based on the intermediate outputs from GBDT and integrate the adversarial learning framework for UDA into the boosting training procedure of GBDT to help it adapt to the target domain. In addition, to fully utilize the unlabelled target data, we apply the pseudo-labelling technique and propose a new instance weighting scheme to reduce the negative impact brought by incorrect pseudo labels. In this paper, we illustrate our solution using LightGBM, a popular implementation of GBDT, but the solution can be easily extended to other GBDT implementations.

In summary, we make the following contributions:

(1) We adapt the adversarial learning framework to train LightGBM for unsupervised domain adaptation.

(2) We propose a new instance weighting scheme for pseudolabelled target data to reduce the negative impact of wrongly labelled target data.

(3) We run experiments on two real large malware datasets to demonstrate the effectiveness of the proposed domain adaptation method. Ablation study is conducted to verify the contribution of each sub-component of the proposed method.

\section{RELATED WORKS}

Domain adaptation techniques have been proposed to deal with the distribution drift issue between the source data and the target data. In this section, we provide a review of the recent domain adaptation works, concerning these three categories: instance-based domain adaptation, feature-based domain adaptation and tree-based domain adaptation.

\subsection{Instance-based Domain Adaptation}

Instance-weighting approaches assign a weight to each source instance to make the reweighted version of the source distribution as similar to the target distribution as possible. Huang et al. [16] proposed a kernel-based method, kernel mean matching (KMM), to reweight instances in a reproducing kernel Hilbert space (RKHS).
Bickel et al. [5] attempted to reweight each source instance in a different way, in which the process of distribution correction is incorporated into a kernelized logistic regression. Loog [18] propounded nearest neighbor weighting $(\mathrm{NNeW})$ scheme, in which nearest neighbor search is used to estimate the mismatch between the densities of source domain and target domain. Zhang et al. [32] studied three situations, target shift, conditional shift, and generalized target shift which combines the above two situations and presented practical approaches to handle them based on the kernel mean embedding of conditional and marginal distributions.

Although the above-mentioned instance-weighting approaches could be easily applied to various domain adaptation tasks, they only perform well when the supports of source distribution and target distribution sufficiently overlap.

\subsection{Feature-based Domain Adaptation}

Feature based domain adaptation approaches aim at transforming source and target data into a common domain-invariant feature space. Daumé III [10] demonstrated a simple kernel-mapping function, which maps the data from both source and target domains to a high-dimensional feature space. Using subspace alignment, Fernando et al. [12] proposed an unsupervised domain adaptation method, in which the subspaces of the source domain and the target domain are created and a linear mapping is learned to align the source subspace with the target one. CORrelation ALignment (CORAL) [24] minimizes the domain shift between source and target distribution by aligning the second-order statistics. More specifically, it transforms the source features using the covariance of the target features, so one of its advantages is that it could be easily applied to any base classifiers. CeHTL [35] is another feature based approach for network attacks detection. It attempts to find the common latent subspace for two domains using linear transformation.

Recent studies have shown that deep neural networks are able to learn more transferable features [30], achieving breakthrough performance on some domain adaptation tasks. One branch is to minimize the distribution distances between two domains during the training of deep learning models. Tzeng et al. [27] introduced adaptation layer and domain confusion loss based on Maximum Mean Discrepancy (MMD) to make the learned feature representation domain-invariant. Then, Zhang et al. [33] proposed Deep Transfer Network (DTN), in which MMD is also adopted to align the marginal distributions and conditional distributions simultaneously. In [29], a weighted MMD based unsupervised domain adaptation method is proposed. Specifically, the weight for each class in the source domain is estimated using EM algorithm so that the class weight bias between source domain and target domain could be reduced. Zellinger et al. [31] introduced Central Moment Discrepancy (CMD), a new distance function between the probability distributions using the higher order central moments information. Compared with MMD, it does not need to calculate the kernel matrix so the computation is much reduced. Similar to [27], Sun and Saenko [25] proposed Deep Coral, which incorporates CORAL into deep learning neural networks by replacing the MMD loss with CORAL Loss. Recently, Chen et al. [6] presented Higher-order Moment Matching (HoMM) method, which unifies 
MMD (first order) and CORAL (second order) into one framework and could be generalized to arbitrary order moment matching.

Another branch is to incorporate adversarial learning into the training of deep learning models. Ganin and Lempitsky [14] proposed domain-adversarial neural network (DANN), in which a domain discriminator is connected to a feature extractor by a novel gradient reversal layer (GRL) so that the learned features are both domain-invariant and discriminative. Such adaptation behavior could be easily achieved in most of the deep learning architectures. Tzeng et al. [26] presented Adversarial Discriminative Domain Adaptation (ADDA), a method that integrates discriminate base model, unshared weights and GAN adversarial loss. Wu et al. [28] proposed to use asymmetrically-relaxed distribution distances in domain-adversarial learning objectives and they provided a theoretical guarantee for target domain performance under assumptions on data distributions. Progressive Feature Alignment Network (PFAN) [7] is another adversarial learning based method, in which an Easy-to-Hard Transfer Strategy (EHTS) and Adaptive Prototype Alignment (APA) are jointly utilized to provide reliable pseudo samples. To preserve both the fine-grained structural information and mode information of the images, Chen and Liu [8] demonstrated STructure-Aware Feature Fusion (STAFF) network based on adversarial game, integrating the mutual information into the learning of domain-invariant features.

Nevertheless, most of the aforementioned deep learning based domain adaptation methods could not be applied to decision tree based machine learning algorithms directly.

\subsection{Tree-based Domain Adaptation}

Several approaches attempt to perform domain adaptation on decision tree based algorithms. TrAdaBoost [9] was proposed to extend AdaBoost for transfer learning under a semi-supervised domain adaptation setting which requires the availability of a small amount of labelled target data. It weakens the impact of the misclassified source instances so that the source distribution would be similar to the target distribution gradually. Different from TrAdaboost, [22] introduced a random forest based semi-supervised domain adaption method in which SER algorithm modifies the structure of each tree through expansion and reduction, while STRUT algorithm fixes the structure and adjusts the threshold in each decision node. A recent work [11] presented the adapted tree boosting algorithm based on XGBoost for building the fraud detection model in secure online transaction. As a semi-supervised domain adaptation method, it utilizes the labelled target data to revise each decision tree in XGBoost through re-split, reweight and special processing to the rare branches.

Most of the existing tree based algorithms are based on tree revising strategies so additional clean labelled target data are in need. In this paper, we propose an unsupervised domain adaptation method that could be easily adopted to existing popular variants of GBDT. Besides, to the best of our knowledge, this is the first domain adaptation approach that integrates adversarial learning into the training of LightGBM, in which there is no explicit revising to the decision trees and the adaptation is accomplished in an adaptive and end-to-end manner.

\section{METHODOLOGY}

\subsection{Problem Definition}

In the unsupervised domain adaptation setting, we denote the sample points i.i.d drawn from the source domain $\mathcal{D}_{s}$ as $\left\{\left(x_{s}^{i}, y_{s}^{i}\right)\right\}_{i=1}^{n_{s}}$, where $x_{s}^{i}$ is the $i_{t h}$ source data sample and $y_{s}^{i}$ is the corresponding label. The samples from the target domain $\mathcal{D}_{t}$ are $\left\{x_{t}^{j}\right\}_{j=1}^{n_{t}}$, whose labels are unknown. The task is to learn a transferable classifier that performs well on the target domain.

\subsection{Preliminary: LightGBM}

LightGBM is a variant of GBDT proposed by Ke et al. [17]. As a state-of-the-art model for malware detection, LightGBM has the advantage of faster computation and higher accuracy. Specifically, LightGBM adopts the leaf-wise tree growth strategy [23] to produce more complex trees, thereby resulting in achieving high accuracy. The scoring function to measure the quality of a tree structure is

$$
\mathbb{S}=\frac{\left[-\operatorname{sgn}\left(\sum_{i \in I} g_{i}\right) \max \left(0,\left|\sum_{i \in I} g_{i}\right|-L_{1}\right)\right]^{2}}{\sum_{i \in I} h_{i}+L_{2}},
$$

where $s g n$ is the sign function; $I$ is the instance set of the current tree structure; $L_{1}$ and $L_{2}$ are the hyper-parameters for L1 and L2 regularization; $g_{i}$ and $h_{i}$ are the first-order gradient and the second-order gradient of the loss w.r.t the output for the $i_{t h}$ instance respectively. The split gain of each possible splitting candidate can then be calculated when constructing each single decision tree based on Equation 1.

\subsection{LightGBM with Adversarial Learning}

The adversarial learning framework in DANN [14] learns the domaininvariant features by connecting a domain discriminator to the feature extractor via gradient reversal layer, so that the domaininvariant features could be gradually generated. However, LightGBM does not generate the features explicitly as deep learning models do. While the outputs of GBDT have been shown to be powerful feature transforms [15], the transformed features are composed of one-hot vectors and thus non-differentiable. To adapt the adversarial learning framework, we construct the features $f$ by concatenating the predictions from all the decision trees, thereby making the features differentiable. We denote the raw output of the LightGBM model in the $r_{t h}$ round as $o_{r} \in \mathbb{R}$; The common way is to use sigmoid function to transform it into the probability, then the prediction is

$$
p_{r}=\operatorname{sigmoid}\left(o_{r}\right)=\frac{1}{1+e^{-o_{r}}} .
$$

Therefore, the feature of the $i_{t h}$ sample $x^{i}$ can be formulated as

$$
f^{i}=G_{f}\left(x^{i}\right)=\left[p_{1}^{i}, \ldots, p_{R}^{i}\right],
$$

where $G_{f}$ is the feature extractor, i.e., the LightGBM, and $R$ is the maximum boosting rounds.

There is another issue in applying adversarial learning in LightGBM. For deep learning models, we can iteratively update the parameters. However, for LightGBM, there are no explicit parameters to update. Therefore, we cannot train the whole LightGBM and the domain discriminator alternatively for multiple iterations. 


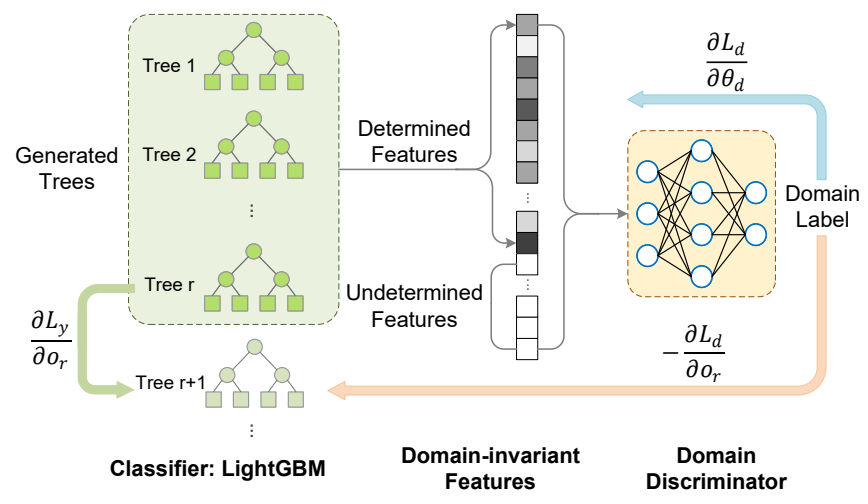

Figure 1: An illustration of LightGBM with adversarial learning

To resolve this issue, we exploit the training algorithm of LightGBM, which trains the trees one by one in a boosting manner. The feedback from the domain discriminator is utilized to train the next decision tree. When training the $r+1_{t h}$ tree, we set $p_{k}^{i}=0$ $(k \in(r, R])$ for Equation 3, as shown in Fig. 1.

We use $G_{d}$ to denote the domain discriminator, a simple Multilayer Perceptron (MLP), whose target is to discriminate the learned features $f$ from source domain and target domain. Thus, the overall optimizing target can be formulated as

$$
L\left(G_{f}, G_{d}\right)=\sum_{i} L_{y}\left(p^{i}, y^{i}\right)-\lambda L_{d}\left(G_{d}\left(G_{f}\left(x^{i}\right)\right), d^{i}\right),
$$

where $L_{y}$ is the classification loss, $L_{d}$ is the domain loss and $\lambda$ is a hyper-parameter to balance these two loss functions. Both of $L_{y}$ and $L_{d}$ are taken as the cross entropy loss function. Besides, $p^{i}$ is the current prediction of LightGBM of the $i_{t h}$ sample, $y^{i}$ is the true class label and $d^{i} \in\{0,1\}$ denotes its domain label, with 0 indicating the source domain and 1 otherwise.

The objective is to find the optimal solution $G_{d}^{*}, G_{f}^{*}$ such that

$$
\begin{aligned}
G_{d}^{*} & =\operatorname{argmax}_{G_{d}} L\left(G_{f}, G_{d}\right), \\
G_{f}^{*} & =\operatorname{argmin}_{G_{f}} L\left(G_{f}, G_{d}\right) .
\end{aligned}
$$

Combining the adversarial learning framework with GBDT is different from with those deep learning based frameworks that couple the feature extractor and the domain discriminator so tightly, so for Equation 5, we train $G_{d}$ for multiple epochs in each boosting round to make sure that its feedback is accurate and reliable enough. For Equation 6, we train the next (i.e., $r+1_{t h}$ ) decision tree of the LightGBM to optimize the loss. As mentioned in Section 3.2, the generation of the $r+1_{t h}$ tree depends on the gradient and hessian produced from the previous $r$ trees. Here, $g_{i}$ and $h_{i}$ of the $i_{t h}$ sample w.r.t the raw output of LightGBM $o_{r}^{i}$ in Equation 1 can be calculated by

$$
\begin{gathered}
g_{i}=\frac{\partial L_{y}^{i}}{\partial o_{r}^{i}}-\lambda \frac{\partial L_{d}^{i}}{\partial o_{r}^{i}}=p_{r}^{i}-y^{i}-\lambda \frac{\partial L_{d}^{i}}{\partial p_{r}^{i}} p_{r}^{i}\left(1-p_{r}^{i}\right), \\
h_{i}=\frac{\partial^{2} L_{y}^{i}}{\partial o_{r}^{i^{2}}}-\lambda \frac{\partial^{2} L_{d}^{i}}{\partial o_{r}^{i^{2}}}=p_{r}^{i}\left(1-p_{r}^{i}\right)\left(1-\lambda \frac{\partial L_{d}^{i}}{\partial p_{r}^{i}}\left(1-2 p_{r}^{i}\right)\right) .
\end{gathered}
$$

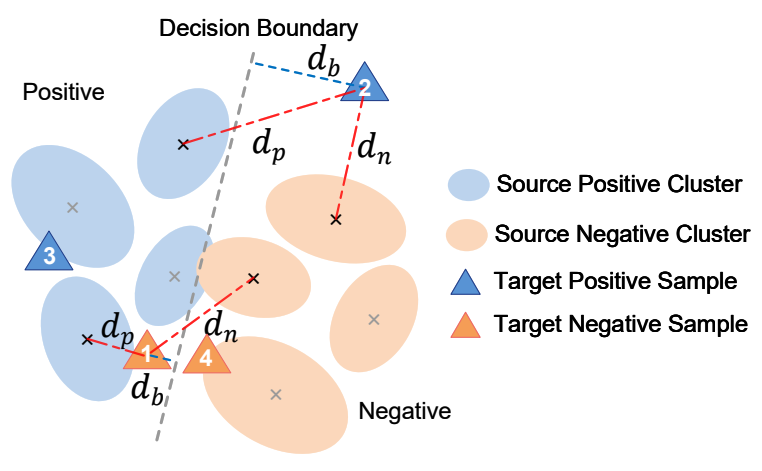

Figure 2: An illustration of instance weighting scheme

As in [14], the domain adaptation hyper-parameter $\lambda$ is initialized as 0 and gradually increased to 1 as the training continues using the schedule: $\lambda=\frac{2}{1+e^{-\gamma \cdot t}}-1$, where $\gamma$ is the temperature constant, and $t \in[0,1]$ is the training progress, so that the negative effects of the noisy signals in the early stage could be alleviated. However, when the classification loss $L_{y}$ is about to converge, the magnitude difference between $L_{y}$ and $L_{d}$ would become very large, which brings degradation to the performance of LightGBM. To address this problem, we design a new schedule for automatically changing $\lambda$ :

$$
\lambda=\left\{\begin{array}{ll}
\frac{2}{1+e^{-\gamma \cdot t}}-1 & t \in\left[0, t_{p}\right] \\
\frac{2}{1+e^{-\gamma \cdot t}}-\frac{2}{1+e^{-\gamma \cdot\left(t-t_{p}\right)}} & t \in\left(t_{p}, 1\right]
\end{array},\right.
$$

where $t_{p}$ is the turning point, i.e., $\lambda$ would be increased before $t_{p}$ and then decreased gradually, which provides a better balance between the classification loss and the domain loss.

\subsection{Instance Weighting Scheme}

We devise a pseudo-labelling technique in order to fully exploit the target data. Specifically, a LightGBM model is first trained using only the source data; then the model assigns a pseudo-label to each target instance.

Next, the source data and the pseudo-labelled target data are merged to train a new LightGBM model. One issue of using the pseudo-labels is that incorrect pseudo-labels could affect the model's performance negatively, similar to noisy data. To mitigate the negative impact of incorrectly predicted target labels while exploiting the advantages of those correctly labelled data, we propose to estimate a weight for each target sample to represent the reliability of the pseudo-label and then sample the target data for training based on the weights.

The gray dash line in Fig. 2 represents the decision boundary of a classifier trained on the source samples to generate the pseudolabels for the target samples. Then the target samples could be categorized into two types:

- Type I with low prediction confidence, e.g., triangle data point 1 and 4 in Fig.2

- Type II with high prediction confidence, e.g., triangle data point 2 and 3 in Fig.2

For Type I data samples, the confidence of the classifier is low, i.e., they are near the decision boundary of the classifier. As their 


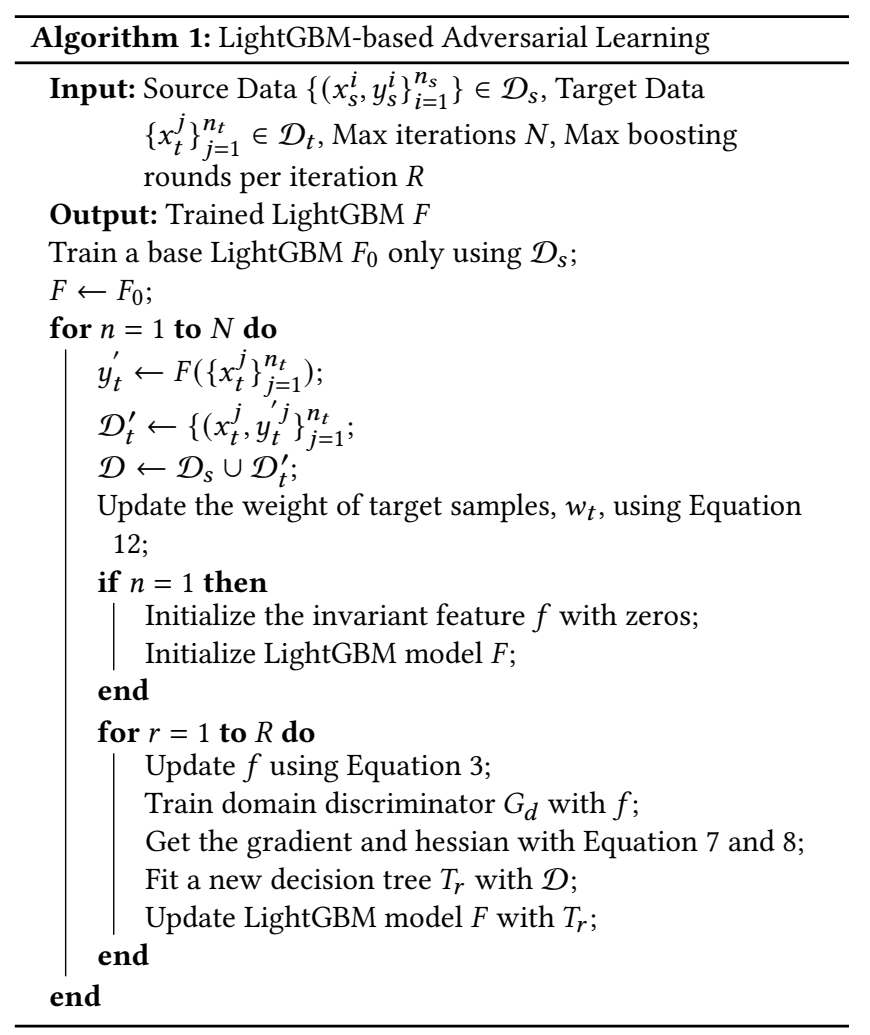

pseudo-labels are likely to be unreliable, a low weight would be assigned to them. We use $d_{b}$ to measure the distance between the data samples and the decision boundary, i.e., the uncertainty:

$$
d_{b}=|1-2 p|,
$$

where $p$ is the probability prediction of the classifier.

For Type II data samples, although the prediction confidence is high, their pseudo labels are also not always trustable depending on the quality of the classifier, i.e., it can still be possible for some of the data samples with high prediction confidence but incorrect pseudo labels (e.g., triangle data point 2). To reduce the impact of these target samples, we first cluster the source samples into $K$ clusters using k-means clustering algorithm for the positive and the negative samples respectively, as shown in Fig. 2. Here we introduce $d_{p}$ (resp. $d_{n}$ ), the distance between a target sample and the centroid of the nearest positive cluster (resp. the negative cluster). If $d_{p}$ and $d_{n}$ are close, the sample is a borderline point between positive and negative clusters. If both $d_{p}$ and $d_{n}$ are also large, the sample is likely to have an incorrectly labelled pseudo-label as it is also far from the positive and negative clusters (e.g., triangle data point 2). Similar to the low prediction confidence points, we assign such samples with low weights. To measure the closeness and the absolute scale of $d_{n}$ and $d_{p}$, we propose the following equation,

$$
d_{c}=\left|\frac{d_{p}-d_{n}}{d_{p}+d_{n}}\right|
$$

Table 1: Summary of the datasets

\begin{tabular}{|c|c|c|c|}
\hline Dataset Name & Data & Positive files & Negative files \\
\hline \multirow{2}{*}{$\begin{array}{c}\text { Private } \\
\text { dataset }\end{array}$} & Apr, 2017 & 58394 & 48595 \\
\cline { 2 - 4 } & May, 2017 & 42635 & 87858 \\
\hline \multirow{3}{*}{$\begin{array}{c}\text { EMBER } \\
\text { dataset }\end{array}$} & Q1, 2018 & 83865 & 73711 \\
\cline { 2 - 4 } & Q2, 2018 & 74201 & 72194 \\
\cline { 2 - 4 } & Q3, 2018 & 85475 & 74140 \\
\cline { 2 - 4 } & Q4, 2018 & 156459 & 129955 \\
\hline
\end{tabular}

By combining these two measures, the weight of each target sample is calculated as

$$
w=\sqrt{\frac{d_{b}^{2}+d_{c}^{2}}{2}} .
$$

The weight provides a basis for sampling the target data in LightGBM. The resulting instance weighting scheme is able to make the most of those correctly predicted samples, for example, the triangle point 3 in Fig. 2. However, some of the correctly labelled samples may face the problem that their $d_{b}$ and $d_{c}$ are small, in which case they will be considered as points with low classifier confidence and also as the borderline points between positive and negative clusters, e.g., the triangle data point 4 . As a result, these points will be assigned low weights, which is a compromise of this weighting scheme. However, it could be alleviated in the next section.

\subsection{Model Training}

We train the LightGBM and conduct pseudo labelling alternatively for $N$ iterations as described in Algorithm 1. In this way, the model and the pseudo labels become more and more accurate. In each iteration, the pseudo labels and the weight of the target data would be updated by the previous model generated in the last iteration. For each boosting round in one iteration, the domain discriminator is trained following the adversarial learning algorithm described in Section 3.3, and then the gradient and hessian of each training sample calculated by Equation 7 and 8 would be utilized to fit the next decision tree. Consequently, the final LightGBM consists of maximum $N \times R$ trees.

With continued training, the wrongly predicted pseudo labels in the early stages of the training could be corrected and be assigned larger weights in time.

\section{EXPERIMENTS}

\subsection{Datasets}

To validate the effectiveness of the proposed method, we conduct experiments on the following two datasets:

- Private dataset This dataset is provided by a local anti-virus company, SecureAge Technology of Singapore, in which a online platform is deployed for continuously collecting PE files from the endpoints and scanning them with 12 anti-virus engines. The scanning results are utilized for labelling each PE file. A sample is labelled as positive if 4 or more engines detect that it is malicious. If none of the engines deems it malicious, then it is labelled as negative. We discard the 
Table 2: Experiment results on Private Dataset

\begin{tabular}{|c|c|c|c|}
\hline \multirow{2}{*}{ Type } & \multirow{2}{*}{ Models } & \multicolumn{2}{|c|}{ April $\rightarrow$ May, 2017 } \\
\cline { 2 - 4 } & & Test Recall $(\%)$ & Test AUC(\%) \\
\hline \multirow{2}{*}{$\begin{array}{c}\text { Traditional } \\
\text { instance based }\end{array}$} & NNeW & $82.77 \pm 0.41$ & $99.79 \pm 0.00$ \\
\cline { 2 - 4 } & KMM & $81.01 \pm 0.39$ & $99.78 \pm 0.00$ \\
\hline \multirow{2}{*}{$\begin{array}{c}\text { Traditional } \\
\text { feature based }\end{array}$} & SUBA & 28.62 & 96.75 \\
\cline { 2 - 4 } & CORAL & 20.25 & 95.57 \\
\cline { 2 - 4 } & CeHTL & $0.06 \pm 0.05$ & $49.99 \pm 0.08$ \\
\hline \multirow{2}{*}{$\begin{array}{c}\text { Deep learning } \\
\text { based }\end{array}$} & CNN Source Only & $12.24 \pm 2.97$ & $89.36 \pm 1.01$ \\
\cline { 2 - 4 } & Deep Coral & $8.11 \pm 3.68$ & $88.06 \pm 2.89$ \\
\cline { 2 - 4 } & DANN & $0.00 \pm 0.00$ & $93.45 \pm 0.91$ \\
\hline \multirow{2}{*}{ Tree based } & LightGBM Source Only & 83.91 & $\mathbf{9 9 . 8 0}$ \\
\cline { 2 - 4 } & Proposed & $\mathbf{8 9 . 7 2} \pm \mathbf{0 . 3 3}$ & $99.77 \pm 0.00$ \\
\hline
\end{tabular}

other inconclusive samples with 1-3 engines labelling them as malicious. All the labelled samples are processed using the feature extraction technique proposed in [1], in which the feature dimension of each PE file is 2351. PE files collected in April (resp. May) form the source (resp. target) domain. The summary of the statistics of this dataset is in Table 1 . Due to the fact that there is no information about the collect date for each PE file, to satisfy the unsupervised domain adaptation setting, the validation data in each experiment is randomly sampled from the source domain.

- EMBER dataset This dataset is a public dataset from EMBER [1], in which PE files are collected in 2018 and archived by month. The feature dimension of each PE file is 2381 . We first divide the dataset into four quarters (Q1, Q2, Q3 and Q4) as displayed in Table 1. To separate the dataset into source and target domains, we process it into six tasks with both single source quarter $(\mathrm{Q} 1 \rightarrow \mathrm{Q} 2, \mathrm{Q} 2 \rightarrow \mathrm{Q} 3$ and Q3 $\rightarrow \mathrm{Q} 4)$ and multiple source quarters $(\mathrm{Q} 1+\mathrm{Q} 2 \rightarrow \mathrm{Q} 3, \mathrm{Q} 2+\mathrm{Q} 3 \rightarrow \mathrm{Q} 4$ and $\mathrm{Q} 1+\mathrm{Q} 2+\mathrm{Q} 3 \rightarrow \mathrm{Q} 4)$. For the experiments of this dataset, we randomly sampled half the data in the last month in the source domain as the validation data.

\subsection{Baseline Methods}

In order to investigate the performance improvement, we compare the proposed model with the following baselines:

- Traditional instance-based methods: NNeW [18] and KMM [16]. Both of them attempt to reweight the source instances to make the distribution of the source domain as similar as the target distribution. For these two baseline models, LightGBM is adopted as the classifier.

- Traditional feature-based methods: SUBA [12], CORAL [24] and CeHTL [35]. They aim to find a common domain-invariant feature space. The transformed features of these two models are fed into LightGBM for classification.

- Deep learning-based methods: For deep-learning based methods, a convolutional neural network $(\mathrm{CNN})$ reads the EMBER feature. Then Deep Coral [25] and DANN [14] are applied for domain adaptation.

- Tree-based method: TrAdaboost [9] is a popular tree based domain adaptation algorithm. However, this algorithm is a

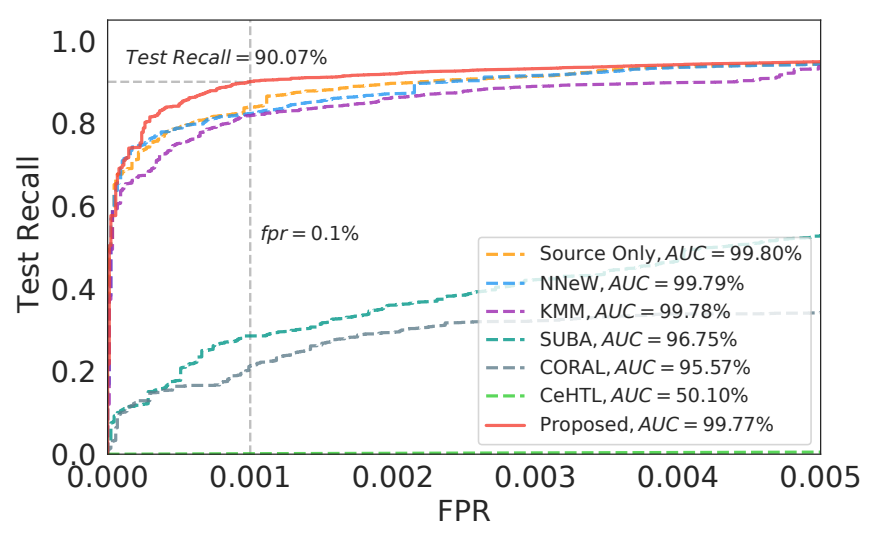

Figure 3: ROC curves of LightGBM based models

semi-supervised domain adaptation method that requires labelled target data for reweighting the source samples, which is different from our unsupervised domain adaptation setting. As mentioned before, for the EMBER dataset, half of the data in the last month of each quarter are randomly picked out as the validation data. Thus, in this experiment, we will utilize another half as the "labelled target data" so that the semi-supervised setting could be satisfied. However, for the private dataset, the specific collection date of each sample in one month is not available, so we are unable to split the data in the source domain to get a "labelled target dataset" with different distribution. Therefore, TrAdaboost is only evaluated on EMBER dataset. For TrAdaboost, the base learner is also LightGBM for fair comparison.

\subsection{Experimental Setup}

The maximum number of estimators of the LightGBM in the baselines is 1000; the early stopping rounds are configured as 50; and the other hyperparameters are set as default. For the proposed model, the hyperparameters of the initial LightGBM $F_{0}$ is configured as the same as those applied in the baselines. For each following iteration, the maximum number of estimators $R$ is 200 , the early stopping rounds are 5 and the total number of iterations $N$ is 5 ; the remaining parameters are set as default. As a result, the final learned LightGBM has the same maximum number of trees as baselines. The domain discriminator in the proposed model is a simple Multilayer Perceptron (MLP) composing of two fully-connected layers $(x \rightarrow 32 \rightarrow 1)$, and only one domain discriminator is trained during the whole learning process. In each boosting round, the maximum epochs of training the domain discriminator is 3 and the early stopping round is set as 1 so that while ensuring the quality of the feedback, the time complexity can also be controlled. In addition, the temperature constant $\gamma$ in adversarial learning is fixed to 10 as in [14], the turning point $t_{p}$ is set to 0.3 and the number of clusters $K$ is 10. $t_{p}$ and $K$ are determined using grid search with the range $[0.2,0.3,0.4,0.5,0.6]$ and $[13,12,11,10,9]$ respectively as Microsoft classifies most malware into 13 categories [19]. For the deep learning-based models, the feature extractor contains one batch normalization layer, three Conv1D layers with kernel size of 4 and 
Table 3: Experiment results on EMBER Dataset (Single Source Quarter)

\begin{tabular}{|c|c|c|c|c|c|c|c|}
\hline \multirow{2}{*}{ Type } & \multirow{2}{*}{ Models } & \multicolumn{2}{|c|}{$\mathrm{Q1} \rightarrow \mathrm{Q} 2$} & \multicolumn{2}{|c|}{$\mathrm{Q} 2 \rightarrow \mathrm{Q3}$} & \multicolumn{2}{|c|}{$\mathbf{Q 3} \rightarrow \mathbf{Q 4}$} \\
\hline & & Test Recall(\%) & Test AUC (\%) & Test Recall(\%) & Test AUC (\%) & Test Recall(\%) & Test AUC(\%) \\
\hline \multirow{2}{*}{$\begin{array}{c}\text { Traditional } \\
\text { instance based }\end{array}$} & $\mathrm{NNeW}$ & $73.96 \pm 0.46$ & $99.28 \pm 0.02$ & $69.04 \pm 0.54$ & $99.21 \pm 0.04$ & $60.57 \pm 0.92$ & $98.86 \pm 0.04$ \\
\hline & KMM & $73.92 \pm 0.73$ & $99.26 \pm 0.01$ & $66.25 \pm 0.44$ & $99.23 \pm 0.01$ & $50.43 \pm 0.60$ & $98.78 \pm 0.01$ \\
\hline \multirow{3}{*}{$\begin{array}{l}\text { Traditional } \\
\text { feature based }\end{array}$} & SUBA & 13.36 & 94.56 & 5.51 & 92.42 & 19.05 & 95.43 \\
\hline & CORAL & 4.49 & 86.81 & 17.21 & 94.36 & 29.98 & 91.80 \\
\hline & CeHTL & $0.07 \pm 0.02$ & $50.00 \pm 0.09$ & $0.03 \pm 0.03$ & $50.13 \pm 0.10$ & $0.06 \pm 0.04$ & $50.05 \pm 0.10$ \\
\hline \multirow{3}{*}{$\begin{array}{c}\text { Deep learning } \\
\text { based }\end{array}$} & CNN Source Only & $11.99 \pm 3.88$ & $86.41 \pm 4.77$ & $0.73 \pm 0.08$ & $55.95 \pm 0.11$ & $10.41 \pm 9.63$ & $90.77 \pm 0.49$ \\
\hline & Deep Coral & $15.98 \pm 3.44$ & $93.90 \pm 0.04$ & $10.42 \pm 2.10$ & $94.68 \pm 0.12$ & $10.06 \pm 9.27$ & $92.12 \pm 0.18$ \\
\hline & DANN & $0.00 \pm 0.00$ & $92.00 \pm 0.56$ & $0.00 \pm 0.00$ & $90.40 \pm 0.11$ & $0.00 \pm 0.00$ & $91.02 \pm 0.79$ \\
\hline \multirow{3}{*}{ Tree based } & LightGBM Source Only & 74.01 & 99.28 & 67.55 & 99.26 & 56.34 & 98.88 \\
\hline & TrAdaboost & 72.58 & 99.23 & 65.31 & 99.25 & 52.11 & 98.84 \\
\hline & Proposed & $78.15 \pm 0.25$ & $99.01 \pm 0.01$ & $74.19 \pm 0.66$ & $99.04 \pm 0.00$ & $67.73 \pm 0.76$ & $98.77 \pm 0.01$ \\
\hline
\end{tabular}

Table 4: Experiment results on EMBER Dataset (Multiple Source Quarters)

\begin{tabular}{|c|c|c|c|c|c|c|c|}
\hline \multirow{2}{*}{ Type } & \multirow{2}{*}{ Models } & \multicolumn{2}{|c|}{$\mathrm{Q1}+\mathrm{Q} 2 \rightarrow \mathrm{Q3}$} & \multicolumn{2}{|c|}{$\mathrm{Q} 2+\mathrm{Q} 3 \rightarrow \mathrm{Q4}$} & \multicolumn{2}{|c|}{$\mathrm{Q} 1+\mathrm{Q} 2+\mathrm{Q} 3 \rightarrow \mathrm{Q4}$} \\
\hline & & Test Recall(\%) & Test AUC $(\%)$ & Test Recall(\%) & Test AUC (\%) & Test Recall(\%) & Test AUC(\%) \\
\hline \multirow{2}{*}{$\begin{array}{c}\text { Traditional } \\
\text { instance based }\end{array}$} & $\mathrm{NNeW}$ & $75.19 \pm 1.18$ & $99.45 \pm 0.01$ & $55.50 \pm 1.80$ & $98.97 \pm 0.02$ & $58.47 \pm 0.34$ & $99.15 \pm 0.01$ \\
\hline & KMM & $72.11 \pm 0.89$ & $99.44 \pm 0.01$ & $46.43 \pm 1.59$ & $98.93 \pm 0.04$ & $54.96 \pm 1.09$ & $99.00 \pm 0.04$ \\
\hline \multirow{3}{*}{$\begin{array}{c}\text { Traditional } \\
\text { feature based }\end{array}$} & SUBA & 6.05 & 89.88 & 10.72 & 92.39 & 5.46 & 91.61 \\
\hline & CORAL & 11.36 & 91.57 & 8.18 & 92.40 & 12.80 & 92.23 \\
\hline & CeHTL & $0.03 \pm 0.03$ & $50.08 \pm 0.08$ & $0.03 \pm 0.05$ & $50.03 \pm 0.06$ & $0.04 \pm 0.05$ & $50.03 \pm 0.03$ \\
\hline \multirow{3}{*}{$\begin{array}{c}\text { Deep learning } \\
\text { based }\end{array}$} & CNN Source Only & $3.96 \pm 4.58$ & $68.17 \pm 17.36$ & $0.78 \pm 0.72$ & $63.35 \pm 15.72$ & $1.38 \pm 1.60$ & $64.08 \pm 16.72$ \\
\hline & Deep Coral & $13.45 \pm 1.71$ & $95.75 \pm 0.18$ & $29.37 \pm 1.18$ & $93.57 \pm 0.34$ & $29.91 \pm 0.88$ & $93.66 \pm 0.22$ \\
\hline & DANN & $0.00 \pm 0.00$ & $89.71 \pm 1.43$ & $0.00 \pm 0.00$ & $88.80 \pm 0.92$ & $0.00 \pm 0.00$ & $90.57 \pm 1.21$ \\
\hline \multirow{3}{*}{ Tree based } & LightGBM Source Only & 73.55 & 99.43 & 50.33 & 98.95 & 52.40 & 98.91 \\
\hline & TrAdaboost & 72.09 & 99.51 & 46.61 & 99.00 & 48.09 & 99.26 \\
\hline & Proposed & $80.95 \pm 0.51$ & $99.30 \pm 0.00$ & $68.12 \pm 0.59$ & $98.43 \pm 0.01$ & $73.37 \pm 1.35$ & $97.97 \pm 0.02$ \\
\hline
\end{tabular}

number of filters of 256, three MaxPooling1D layers with kernel size of 4 and one GlobalMaxPooling1D layer; the classifier is also composed of two fully-connected layers; the domain discriminator in DANN is of the same structure as the classifier.

Each experiment is repeated for three times since some of the evaluated methods depend on randomness and we report the mean and the standard variation. Two evaluation metrics are considered to measure the performance of the proposed model and the baseline models: ROC AUC score and Recall (true positive rate) when FPR (false positive rate) is $0.1 \%$. Recall and FPR are defined by:

$$
\begin{aligned}
& \text { Recall }=\frac{t p}{t p+f n}, \\
& F P R=\frac{f p}{f p+t n},
\end{aligned}
$$

where $t p, f p, t n, f n$ are the true-positives, false-positives, truenegatives and false-negatives counts respectively.

For the malware detection task, Recall refers to the ratio of the number of correctly detected malware over all malware. Malware detection models are expected to achieve high recall while generating few false alarms (FPR, usually less than $0.1 \%$ [21]). The Test Recall reported in this paper measures this metric exactly; therefore, we will mainly compare our method with the baselines using this Test Recall.

\subsection{Results and Analysis}

Table 2 provides the summary of the experiment results on the private dataset while Table 3 and Table 4 are the results on EMBER dataset with single source quarter and multiple source quarters. The results show that the proposed method achieves both the best Test Recall on private dataset and EMBER dataset among all the baselines, confirming the effectiveness of our method in detecting new and constantly evolving malware even under a low FPR $(0.1 \%)$. In particular, for the task Q2 $+\mathrm{Q} 3 \rightarrow \mathrm{Q} 4$ in Table 4 , the proposed model outperforms the LightGBM model trained with only source data by $17.79 \%$ and the baseline model, NNeW, by $12.62 \%$ in Test Recall. However, the AUC score of the proposed model is slightly lower than that of LightGBM with only the source data. Fig. 3 displays the ROC curve of those models with LightGBM as the classifier. For some of the baselines, the performance might be good when FPR is high, so that the overall AUC score might be slightly larger. However, when FPR is becoming small, the recall degrades very rapidly.

From the results, traditional instance based methods, NNeW and KMM, achieved comparable performance with LightGBM trained with only source data. However, the performance improvement is marginal and sometimes they face performance degradation after instance reweighting. For the traditional feature-based models, 


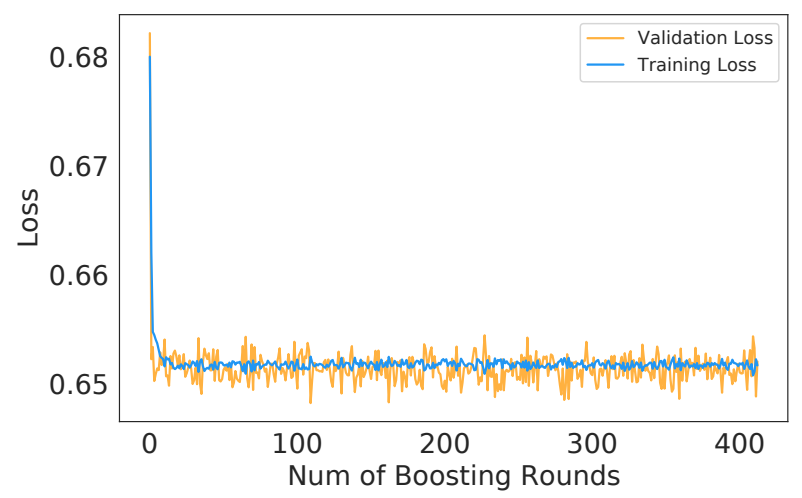

(a) Loss Curves

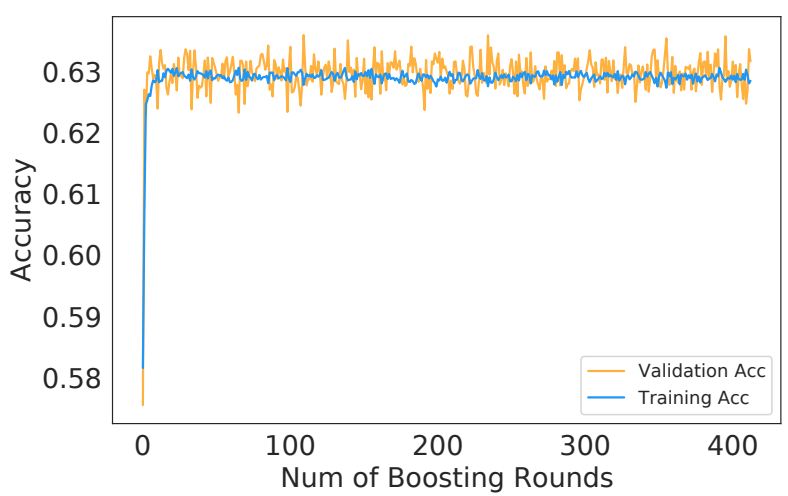

(b) Accuracy Curves

Figure 4: Leaning Curves of Domain Discriminator

SUBA and CORAL, the performance is not good even with LightGBM as the classifier, which implies that it might not be suitable to map the heterogeneous EMBER features into domain-invariant feature space directly without taking the classification performance into consideration. The performance of the three DNN models verifies our assumption that deep learning models are not suitable for processing heterogeneous features including the EMBER features. The Test Recalls are extremely low for the three deep learning models. This is because they make predictions with high confidence and the threshold for $0.1 \%$ FPR becomes extremely high, even to 1. As for the tree-based methods, TrAdaboost is outperformed by LightGBM trained with only source data, which might be due to the unavailability of real "labelled target data".

Some other interesting conclusions could also be drawn from Table 3 and Table 4. As the source domain expands from single quarter to multiple quarters, the amount of data increases, but it also brings data whose distribution are more different from the target domain. Thus, each model may react differently to such changes depending on its design and focus.

As mentioned, the instance based models (NNeW and KMM) perform well only when the distributions of the source and target domain are sufficiently similar, so they are sensitive to the distribution changes. When the target domain is Q4, after combining the data from Q2 with Q3, the performance of these models degrades sharply, which implies that the distributions of Q2 and Q4 differ greatly. However, from the comparison of Q2 $\rightarrow$ Q3 and Q1 + Q2 $\rightarrow$ Q3, the performance of most of the models is improved by a large margin, so different from Q2 and Q4, the distributions of Q1 and Q3 seem to be more similar. Another supporting evidence comes from the comparison of Q2+Q3 $\rightarrow$ Q4 and Q1 + Q2 + Q3 $\rightarrow$ Q4, except for SUBA, all the other models achieve better performance. Thus, the addition of Q1 brings richer data for Q3 and alleviates the negative impact of Q2 on Q4 to some extent.

The feature-based models including the traditional and deep learning based models aim at transforming the features of source domain and target domain into domain-invariant features. Therefore, if the distribution of the source and target domains differ widely, it will inevitably increase the difficulty of the transformation. However, some of the feature based models are relatively robust against the distribution changes, e.g., for Deep Coral, from Q3 $\rightarrow$ Q4 to Q2 + Q3 $\rightarrow$ Q4, its Test Recall is increased from $10.06 \%$ to $29.37 \%$, which confirms the ability of deep neural networks to learn more transferable features. However, limited by the weakness of deep learning models in processing the heterogeneous feature, its performance is still unacceptable in these tasks.

For the tree-based baseline models, LightGBM with source only and TrAdaboost face the similar performance degradation caused by significant distribution changes (e.g., Q3 $\rightarrow$ Q4 to Q2 + Q3 $\rightarrow$ Q4). TrAdaboost depends on the "labelled target data" to reweight the source samples. Essentially, it is also an instance-weighting method, and the mentioned limitation for the instance based methods also exists. However, when the difference between the source distributions is negligible (Q2 $\rightarrow$ Q3 to Q1 + Q2 $\rightarrow$ Q3), the performance has been improved by benefiting from the increase in data volume.

It should be noted that the proposed model is robust against the distribution changes. Even when the source domain changes from Q3 to Q2 + Q3, the performance on the target domain Q4 still gets improved, i.e., Test Recall is increased from $67.73 \%$ to $68.12 \%$, confirming that the proposed model is capable of learning domaininvariant features through the designed adversarial learning scheme and making use of the given data as much as possible. With its powerful and robust capabilities, we have improved the Recall on Q3 to $80.95 \%$, and the Recall on Q4 to $73.37 \%$.

As the proposed method involves the adversarial learning framework, we provide the learning curves of the domain discriminator in Fig. 4 by recording its loss and the accuracy with the best validation performance in each boosting round. From Fig. 4(a), the domain discriminator converges fast within the first several rounds, and then the domain discriminator and the feature generator reach a good balance.

\subsection{Ablation Study}

4.5.1 Effect of each component in the proposed method. The proposed method consists of several components, including adversarial 



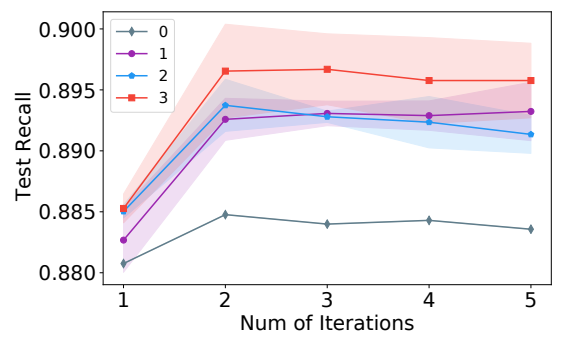

(a) Different Components

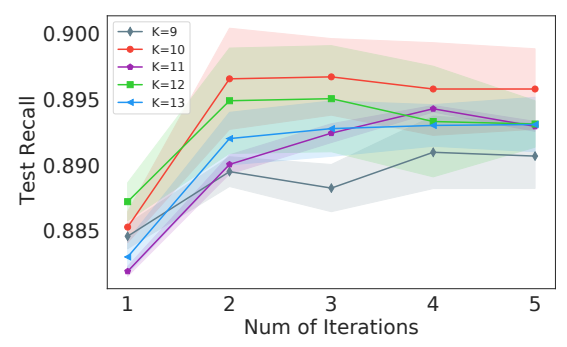

(b) Different $\mathrm{K}$

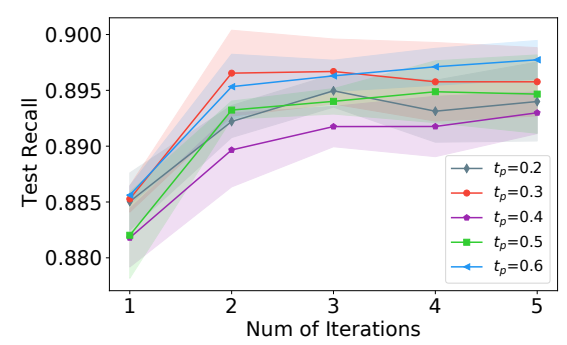

(c) Different $t_{p}$

Figure 5: Comparison of Validation (Upper) and Test (lower) Performance with different configurations

learning in LightGBM and the instance weighting scheme. To explore the effects of different configurations, we conduct comparison experiments with the settings displayed in Table 5. For all these four settings, the unlabelled target data is utilized and the LightGBM model is trained in a continue training way, i.e., the pseudo-labels of the target data are updated per iteration.

Table 5: Different Component Settings

\begin{tabular}{|c|c|c|}
\hline Setting & Adversarial Learning & Weighting scheme \\
\hline 0 & No & No \\
\hline 1 & No & Yes \\
\hline 2 & Yes & No \\
\hline 3 & Yes & Yes \\
\hline
\end{tabular}

Fig. 5(a) depicts the comparisons of these four settings. We can see that setting 0 achieves a comparable validation performance with setting 2, but is slightly worse than setting 1 . Although these three curves tend to be closer to at later iterations, the curve of setting 2 shows slightly better performance. From the curves of the Test Recall, both adversarial learning (setting 2) and weighting scheme (setting 1) improve the test performance over setting 0 on target dataset by a large margin, which corroborates that the adversarial learning is capable of learning domain-invariant features and the weighting scheme is able to effectively alleviate the negative effects of the incorrectly labelled target data and enhance the domain adaptation performance. The combination of these two methods (setting 3) achieve the best performance consistently on the validation data and test data, which indicates that these two methods also complemented each other to promote the performance.

4.5.2 Effect of different $K$, the number of the clusters. Grid search is conducted for finding the best hyperparameter combination of the number of clusters $K$ and the turning point $t_{p}$. Here we display the comparison of different $K \in[13,12,11,10,9]$ with the same best turning point $t_{p}=0.3$ in Fig. $5(\mathrm{~b}) . K=10$ has the best performance on both the validation and test datasets. As $K$ increases, the model encounters a performance decay (e.g., $K=13$ ), indicating that with too many clusters, the difference between $d_{n}$ and $d_{p}$ are narrowed, which weakens the effect of the proposed weighting scheme.

4.5.3 Effect of different $t_{p}$, the turning point. The comparison of different $t_{p}$ is demonstrated in Fig. 5(c). Here we fix the $K$ as 10 , and tune $t_{p}$ from 0.2 to 0.6 . The best validation performance is achieved with $t_{p}=0.3$. For the test dataset, $t_{p}=0.6$ has better performance at the last few iterations. However, it is not suitable to take the test performance to tune the hyperparameters, since the labels of the test data are not available in practice (after deployment). Thus, we chose $t_{p}=0.3$ as the best turning point.

\section{CONCLUSION}

In this paper, we have proposed a novel unsupervised domain adaptation method for static malware detection based on LightGBM. Specifically, we have incorporated adversarial learning into the training of LightGBM to help LightGBM learn domain-invariant features, and designed a new instance weighting scheme to alleviate the negative effects from incorrectly labelled target data during pseudo labelling. The proposed method could be extended to other variants of GBDT algorithms, e.g., XGBoost, etc. Experiments on two real large malware datasets demonstrated the superiority and the robustness of our proposed method over other baselines.

\section{ACKNOWLEDGMENTS}

This research is supported by the National Research Foundation, Prime Ministers Office, Singapore under its National Cybersecurity R\&D Programme (Grant No. NRF2016NCR-NCR002-020). We also thank SecureAge Technology of Singapore for providing the data. 


\section{REFERENCES}

[1] Hyrum S Anderson and Phil Roth. 2018. Ember: an open dataset for training static pe malware machine learning models. arXiv preprint arXiv:1804.04637 (2018).

[2] Andrew Arnold, Ramesh Nallapati, and William W Cohen. 2007. A comparative study of methods for transductive transfer learning. In Seventh IEEE ICDMW 2007. IEEE, 77-82.

[3] AV-TEST. 2017. Security Report 2017/18. https://www.av test.org/fileadmin/pdf/security_report/AV-TEST_Security_Report_2017-2018.pdf (2017).

[4] Banco Santander. 2019. Santander Customer Transaction Prediction https://www.kaggle.com/c/santander-customer-transaction-prediction (2019).

[5] Steffen Bickel, Michael Brückner, and Tobias Scheffer. 2009. Discriminative learning under covariate shift. Fournal of Machine Learning Research 10, 9 (2009).

[6] Chao Chen, Zhihang Fu, Zhihong Chen, Sheng Jin, Zhaowei Cheng, Xinyu Jin, and Xian-Sheng Hua. 2020. HoMM: Higher-Order Moment Matching for Unsupervised Domain Adaptation. Proceedings of AAAI 202034 (04 2020), 3422 3429. https://doi.org/10.1609/aaai.v34i04.5745

[7] Chaoqi Chen, Weiping Xie, Wenbing Huang, Yu Rong, Xinghao Ding, Yue Huang, Tingyang Xu, and Junzhou Huang. 2019. Progressive feature alignment for unsupervised domain adaptation. In CVPR. 627-636.

[8] Qingchao Chen and Yang Liu. 2020. Structure-Aware Feature Fusion for Unsupervised Domain Adaptation.. In AAAI. 10567-10574.

[9] Wenyuan Dai, Qiang Yang, Gui-Rong Xue, and Yong Yu. 2007. Boosting for transfer learning. In ICML. 193-200.

[10] Hal Daumé III. 2009. Frustratingly easy domain adaptation. arXiv preprint arXiv:0907.1815 (2009)

[11] Wenjing Fang, Chaochao Chen, Bowen Song, Li Wang, Jun Zhou, and Kenny Q Zhu. 2019. Adapted tree boosting for Transfer Learning. In IEEE International Conference on Big Data. IEEE, 741-750.

[12] Basura Fernando, Amaury Habrard, Marc Sebban, and Tinne Tuytelaars. 2013. Unsupervised visual domain adaptation using subspace alignment. In Proceedings of ICCV. 2960-2967.

[13] Jerome H Friedman. 2001. Greedy function approximation: a gradient boosting machine. Annals of statistics (2001), 1189-1232.

[14] Yaroslav Ganin and Victor Lempitsky. 2015. Unsupervised domain adaptation by backpropagation. In ICML. 1180-1189.

[15] Xinran He, Junfeng Pan, Ou Jin, Tianbing Xu, Bo Liu, Tao Xu, Yanxin Shi, Antoine Atallah, Ralf Herbrich, Stuart Bowers, et al. 2014. Practical lessons from predicting clicks on ads at facebook. In Proceedings of the Eighth International Workshop on Data Mining for Online Advertising. 1-9.

[16] Jiayuan Huang, Arthur Gretton, Karsten Borgwardt, Bernhard Schölkopf, and Alex J Smola. 2007. Correcting sample selection bias by unlabeled data. In Advances in neural information processing systems. 601-608.

[17] Guolin Ke, Qi Meng, Thomas Finley, Taifeng Wang, Wei Chen, Weidong Ma Qiwei Ye, and Tie-Yan Liu. 2017. Lightgbm: A highly efficient gradient boosting decision tree. In NeurIPS. 3146-3154.

[18] Marco Loog. 2012. Nearest neighbor-based importance weighting. In 2012 IEEE International Workshop on Machine Learning for Signal Processing. IEEE, 1-6.
[19] Microsoft. 2020. How Microsoft identifies malware and potentially unwanted applications. https://docs.microsoft.com/en-us/windows/security/threatprotection/intelligence/ (2020).

[20] Porto Seguro. 2017 Porto Seguro's Safe Driver Prediction. https://www.kaggle.com/c/porto-seguro-safe-driver-prediction (2017).

[21] Joshua Saxe and Konstantin Berlin. 2015. Deep Neural Network Based Malware Detection Using Two Dimensional Binary Program Features. In CoRR abs/1508.03096. https://doi.org/10.1109/MALWARE.2015.7413680 arXiv:1508.03096

[22] Noam Segev, Maayan Harel, Shie Mannor, Koby Crammer, and Ran El-Yaniv. 2016. Learn on source, refine on target: A model transfer learning framework with random forests. IEEE transactions on pattern analysis and machine intelligence 39 , 9 (2016), 1811-1824.

[23] Haijian Shi. 2007. Best-first decision tree learning. Ph.D. Dissertation. The University of Waikato.

[24] Baochen Sun, Jiashi Feng, and Kate Saenko. 2015. Return of frustratingly easy domain adaptation. arXiv preprint arXiv:1511.05547 (2015).

[25] Baochen Sun and Kate Saenko. 2016. Deep coral: Correlation alignment for deep domain adaptation. In ECCV. Springer, 443-450.

[26] Eric Tzeng, Judy Hoffman, Kate Saenko, and Trevor Darrell. 2017. Adversarial discriminative domain adaptation. In CVPR. 7167-7176.

[27] Eric Tzeng, Judy Hoffman, Ning Zhang, Kate Saenko, and Trevor Darrell. 2014. Deep domain confusion: Maximizing for domain invariance. arXiv preprint arXiv:1412.3474 (2014).

[28] Yifan Wu, Ezra Winston, Divyansh Kaushik, and Zachary Lipton. 2019. Domain adaptation with asymmetrically-relaxed distribution alignment. arXiv preprint arXiv:1903.01689 (2019)

[29] Hongliang Yan, Yukang Ding, Peihua Li, Qilong Wang, Yong Xu, and Wangmeng Zuo. 2017. Mind the class weight bias: Weighted maximum mean discrepancy for unsupervised domain adaptation. In CVPR. 2272-2281.

[30] Jason Yosinski, Jeff Clune, Yoshua Bengio, and Hod Lipson. 2014. How transferable are features in deep neural networks? In NeurIPS, Z. Ghahramani, M. Welling, C. Cortes, N. D. Lawrence, and K. Q. Weinberger (Eds.). Curran Associates, Inc., 3320-3328. http://papers.nips.cc/paper/5347-how-transferable-are-features-indeep-neural-networks.pdf

[31] Werner Zellinger, Thomas Grubinger, Edwin Lughofer, Thomas Natschläger, and Susanne Saminger-Platz. 2017. Central moment discrepancy (cmd) for domaininvariant representation learning. arXiv preprint arXiv:1702.08811 (2017).

[32] Kun Zhang, Bernhard Schölkopf, Krikamol Muandet, and Zhikun Wang. 2013. Domain adaptation under target and conditional shift. In International Conference on Machine Learning. 819-827.

[33] Xu Zhang, Felix Xinnan Yu, Shih-Fu Chang, and Shengjin Wang. 2015. Deep transfer network: Unsupervised domain adaptation. arXiv preprint arXiv:1503.00591 (2015).

[34] Zhaoqi Zhang, Panpan Qi, and Wei Wang. 2020. Dynamic Malware Analysis with Feature Engineering and Feature Learning. In Proceedings of the AAAI 2020, Vol. 34. 1210-1217.

[35] Juan Zhao, Sachin Shetty, Jan Wei Pan, Charles Kamhoua, and Kevin Kwiat. 2019. Transfer learning for detecting unknown network attacks. EURASIP fournal on Information Security 2019, 1 (2019), 1. 